\title{
BMJ Open Increasing the uptake of long-acting reversible contraception in general practice: the Australian Contraceptive ChOice pRoject (ACCORd) cluster randomised controlled trial longitudinal follow-up protocol
}

\author{
Danielle Mazza, ${ }^{1}$ Natalie Amos (D) , ${ }^{1}$ Cathy J Watson (D) , ${ }^{1}$ Kevin McGeechan, ${ }^{2}$ \\ Marion Haas, ${ }^{3}$ Jeffrey F Peipert, ${ }^{4}$ Jayne Lucke, ${ }^{5,6}$ Angela Taft, ${ }^{7}$ \\ Kathleen McNamee, ${ }^{8}$ Kirsten I Black ${ }^{9}$
}

To cite: Mazza D, Amos N, Watson CJ, et al. Increasing the uptake of long-acting reversible contraception in general practice: the Australian Contraceptive ChOice pRoject (ACCORd) cluster randomised controlled trial longitudinal follow-up protocol. BMJ Open 2020;10:e035895. doi:10.1136/ bmjopen-2019-035895

- Prepublication history for this paper is available online. To view these files, please visit the journal online (http://dx.doi org/10.1136/bmjopen-2019035895).

Received 29 November 2019 Revised 30 March 2020 Accepted 29 May 2020

Check for updates

(C) Author(s) (or their employer(s)) 2020. Re-use permitted under CC BY-NC. No commercial re-use. See rights and permissions. Published by BMJ.

For numbered affiliations see end of article.

Correspondence to Professor Danielle Mazza; Danielle.Mazza@monash.edu

\section{ABSTRACT}

Introduction Through addressing main barriers to the uptake of long-acting reversible contraceptives (LARCs) among Australian women, the Australian Contraceptive ChOice pRoject (ACCORd) trialled an educational intervention targeting general practitioners (GPs) and provided those in the intervention group with a rapid referral service for quick insertion. The cluster randomised controlled trial resulted in greater uptake of LARC in the intervention group. This protocol paper describes a longitudinal follow-up to the ACCORd Study to assess the long-term efficacy and cost-effectiveness of the intervention.

Methods and analysis Women participants (patients of ACCORd GPs) completed a baseline, 6 -month and 12-month survey. These participants will be invited to complete an additional follow-up survey 3 years post completion of their baseline interview. Based on the original ACCORd Study tools, the online survey will address long-term outcomes including contraceptive continuation rates and reproductive history, any unintended pregnancies, satisfaction and concerns with their current contraceptive method, and an assessment of quality of life. We will analyse data using binary regression models with generalised estimating equations and robust standard errors to account for clustering.

Discussion Demonstration of sustained use, effectiveness at reducing unwanted pregnancies and cost-effectiveness of this strategy among this cohort of Australian primary care patients, will strengthen the policy and programme urgency of addressing wider dissemination of these strategies and replicating the study elsewhere.

Ethics and dissemination The ACCORd Study received approval from the Monash University Human Research Ethics Committee: CF16/188-201000080. Additionally, an amendment to conduct this 3-year longitudinal follow-up survey has been approved. The trial follow-up outcomes will be disseminated through formal academic pathways, including journal articles, national and international conferences and reports as well as using more
Strengths and limitations of this study

- The Australian Contraceptive ChOice pRoject (ACCORd) successfully trialled a complex practicebased intervention to improve the uptake of longacting reversible contraception among Australian women.

- A longitudinal 3-year follow-up of the ACCORd Study will be conducted via an online survey to assess the long-term acceptability, effectiveness and costeffectiveness of the intervention.

- The trial was conducted in metropolitan Melbourne; consequently, the participating general practitioners and women may not be representative of the broader Australian population.

'mainstream' strategies such as seminars, workshops and media engagement. Additionally, outcomes will be communicated through policy briefs to Australian state and federal governments.

Trail registration number This trial is registered with the Australian and New Zealand Trials Registry ACTRN12615001346561. Recruitment and data collection have been completed for the baseline, 6 -month and 12-month surveys. Data collection for the 3-year survey commenced in August 2019.

\section{INTRODUCTION}

In an effort to reduce rates of unintended pregnancies in Australia, the Australian Healthcare and Hospitals Association has expressed the need for more research into the barriers as well as the facilitators of long-acting reversible contraceptive (LARC) uptake, including intrauterine devices and implants. ${ }^{1}$ The Australian Contraceptive ChOice pRoject (ACCORd) ${ }^{23}$ is an Australian 
adaptation of the successful Contraceptive CHOICE Project (CHOICE) ${ }^{4}$ conducted in the USA, and is the first study in Australia to evaluate whether a complex intervention in general practice settings resulted in increased LARC uptake among women. The ACCORd intervention involved training general practitioner (GPs) to deliver efficacy-focused contraceptive counselling as well as providing a rapid referral pathway for insertion of LARCs. In the control group, GPs provided usual care contraceptive counselling; they did not receive any training and were not given access to a rapid referral pathway for LARC insertion. The ACCORd Study aimed to address patient, practitioner and system barriers in order to increase the uptake of LARCs and reduce rates of unintended pregnancy.

The ACCORd intervention resulted in increased LARC uptake among women. Within 4 weeks of receiving contraceptive counselling during a GP consultation, 19.3\% of women in the intervention group had an LARC inserted, compared with $12.9 \%$ in the control group. At 6 months and 12 months post baseline, a significantly greater proportion of participants in the intervention group were using an LARC compared with the control group. Notably, no differential effects were found for age, parity, use of LARC at baseline, marital status, socioeconomic status, education, previous unintended pregnancy or previous abortion. ${ }^{2}$

While the findings of the ACCORd Study are promising, the 6-month and 12-month follow-up timeline provides little insight into the long-term sustained impact of the intervention. In the US CHOICE Study, a follow-up survey was conducted every 6 months up to 3 years post enrolment. More than 9000 women participated in the CHOICE Study and the intervention resulted in a significant increase in the uptake of LARCs. At 12-month and 24-month LARC users reported greater continuation rates of their chosen contraceptive method $(87 \%$ at 12 months, $77 \%$ at 24 months) than non-LARC users (57\% at 12 months, $41 \%$ at 24 months). ${ }^{5}$ Consequently, the researchers observed a reduction in teen pregnancy, birth and abortion in the St Louis region, (the site of the CHOICE Study), compared with the national rates among sexually active teens. ${ }^{6}$ At 3 years, the CHOICE Study again found impressive continuation rates for LARC methods $(67.2 \%)$ compared with non-LARC methods $(31 \%){ }^{7}$

We are yet to examine similar longitudinal data in the Australian context. While the ACCORd Study has not involved 6-monthly follow-ups, a longitudinal follow-up (3years from baseline) of the participants of the ACCORd Study will provide an understanding of the longer-term outcomes of the ACCORd intervention. This follow-up study will further evaluate the effectiveness and cost-effectiveness of the intervention in increasing LARC uptake, sustaining its use and decreasing unplanned pregnancies. Specifically, the longitudinal follow-up will examine the continuation rate of contraceptive method chosen, particularly comparing LARC to non-LARC methods; current contraceptive methods used by women in the intervention compared with the control group; and a comparison of incidents of unintended pregnancy and abortion since the previous survey between LARC and non-LARC users. In addition, a cost-effectiveness evaluation of the intervention will be conducted.

\section{METHODS AND TRIAL DESIGN The ACCORd Study}

The ACCORd Trial was a cluster randomised controlled trial set in metropolitan Melbourne, Australia, using GPs as the unit of randomisation. Twenty-five intervention GPs and thirty-two control GPs participated in the study. Only one GP per clinic was included in the study in order to avoid contamination due to cross-over effects and the clinic reception staff assisted with recruiting the patient participants.

Women attending the clinic were invited by the reception staff to complete an online eligibility survey on an iPad in the clinic waiting room. Women were eligible to participate if they were between 16 and 45 years old, had been sexually active with a male partner in the previous 6 months or anticipated sexual activity in the following 6 months, had not undergone tubal ligation or hysterectomy, had sexual partners who had not undergone a vasectomy, were neither pregnant or anticipating a pregnancy in the following 12 months, spoke proficient English and were interested in discussing contraception or in starting a new, reversible contraceptive method.

Contact details were recorded with the eligibility survey and all eligible women were contacted by phone by an ACCORd researcher to obtain consent and complete baseline questionnaires. Women were asked to make an appointment with their ACCORd GP for contraceptive counselling within 1 week of their enrolment. Additional charges for these appointments were covered by ACCORd to ensure that the participants did not bear any out-of-pocket expenses for the additional visit.

The GPs who were allocated to the intervention group underwent training to deliver structured contraceptive counselling $^{8}$ consisting of non-biassed, scripted descriptions of all available contraceptive methods, with a particular focus on the efficacy and safety of each method. The intervention GPs were also given access to rapid referral LARC insertion clinics via an online booking system.

The intervention GPs delivered the structured contraceptive counselling to the participating women as well as collected clinical information from the women to identify any contraindications or conditions that may influence the choice of contraception. Women were then able to choose their preferred method, provided that it was not medically contraindicated. The GP was also advised to screen for pregnancy, including history and a urine test, and for Chlamydia trachomatis (according to clinical practice guidelines published by the Royal Australian College of General Practitioners). ${ }^{9}$

In the instance where a woman chose an LARC method, intervention GPs could book an appointment for the 
woman via the online rapid referral system to an LARC insertion clinic with a local gynaecologist. Two gynaecologist practices were involved in the study. One of the gynaecologists was female and charged fees for insertion; the other was male and charged no out-of-pocket expenses.

The GPs in the control group were required to provide usual care to women allocated to this group. The control group was neither given access to the educational intervention nor to the rapid referral LARC insertion clinics.

Women were eligible to participate in the ACCORd Study if they were 16-45 years old, had been sexually active with a male partner in the previous 6 months or anticipated sexual activity in the following 6 months, had not undergone tubal ligation or hysterectomy, had sexual partners who had not undergone vasectomy, were neither pregnant nor anticipating a pregnancy in the next 12 months, spoke proficient English and were interested in discussing contraception or in starting a new reversible contraceptive method. Participating women were asked to complete follow-up surveys online at 6 and 12 months after enrolment in the study. Baseline, 6-month and 12-month surveys involved a questionnaire adapted from the US Contraceptive CHOICE Project (CHOICE) ${ }^{5}$ and included the Health Literacy Questionnaire ${ }^{10}$ at baseline and 6 months, and Medical Outcomes Survey $\left(\right.$ SF-36) ${ }^{11}$ at baseline, 6 and 12 months.

\section{ACCORd longitudinal study for the 3-year follow-up}

\section{Recruitment}

Women who participated in the original ACCORd Study will be invited to complete an additional online survey as close as possible to the 3-year anniversary of completing their original baseline assessment. Additional consent will be obtained via an online form at the beginning of the online survey, following an explanatory statement.

Participants will be emailed with an invitation to take part in the ACCORd 3-year follow-up survey. The email will include a link to the Research Electronic Data Capture (REDCap) Survey. A follow-up phone call will be made to confirm receipt of the email and to gently remind women to complete the survey if they wish to, additionally providing the option to complete the survey as a telephone interview. Text message reminders will also be sent if women are yet to complete the survey. Compensation for time of $\$ 30$ in the form of an online gift card will be offered to women for completing the survey. Participants will be recruited for the 3-year follow-up survey from August 2019 until August 2020.

\section{Sample size}

A total of 740 women participated in the ACCORd Study. For the 3-year follow-up we will be inviting all participants who completed the baseline survey and did not actively withdraw from the study $(\mathrm{n}=705)$. Based on a $71 \%$ response rate at both the 6-month and 12-month follow-up, a similar response rate for the 3-year follow-up is expected.

\section{Outcome measures}

The 3-year survey will involve an adapted version of the ACCORd 12-month survey, as well as the SF-36 to measure participants' quality of life. During the survey, women will be asked to report on their current contraceptive use as well as providing an indication of their satisfaction with the contraceptive method on a 3-point Likert scale ranging from 'not satisfied' to 'very satisfied.' Women will also be asked to provide a reproductive history and respond to questions in relation to each pregnancy that they have experienced since the previous survey, including an item on whether or not the pregnancy was unintended. Figure 1 describes the variables recorded throughout the trial to date as well as the proposed 3-year follow-up.

The primary outcome for this longitudinal follow-up is the continuation rate of the use of LARCs compared with non-LARCs; secondary outcomes include the current contraceptive method used; satisfaction with contraceptive choice, the number of unintended pregnancies and quality of life. Other data collected (see figure 1) will be used to investigate mediating variables.

\section{Economic evaluation}

The additional longitudinal data will be used to extend the economic evaluation of the original 6-month and 12-month data. An economic evaluation of the original data is underway, comparing the intervention and control groups in a cost-effectiveness analysis (CEA). The perspective used for this analysis is that of the healthcare system. Costs include the design and implementation of the GP education tool, direct and indirect costs of health service use, and the purchase and administration of contraceptive products. The outcomes for the economic evaluation will be measured in terms of number of LARCs inserted, type of contraception chosen, health-related quality of life (HRQoL) and quality-adjusted life years (QALYs) gained. HRQoL is measured using the 36-Item Short Form survey (SF36) and QALYs is estimated using the Short Form Six-Dimension health index (SF6D), which allows the calculation of QALYs from the SF36 for use in the CEA.

\section{Patient and public involvement}

Patients were not involved in the consultative process for the study design of the ACCORd Study.

\section{Analyses}

Description of groups

We will summarise the sociodemographic (age (16-24, 25-34, 35-44 years), marital status (single, married/ de facto), income (below or above $\$ 600$ per week) and education (completed year 12 or not)) and reproductive history (parity, previous abortion, previous unintended pregnancy, use of LARC at baseline) characteristics of the women from both the intervention and control groups who complete the 3-year survey, with counts and proportions. 


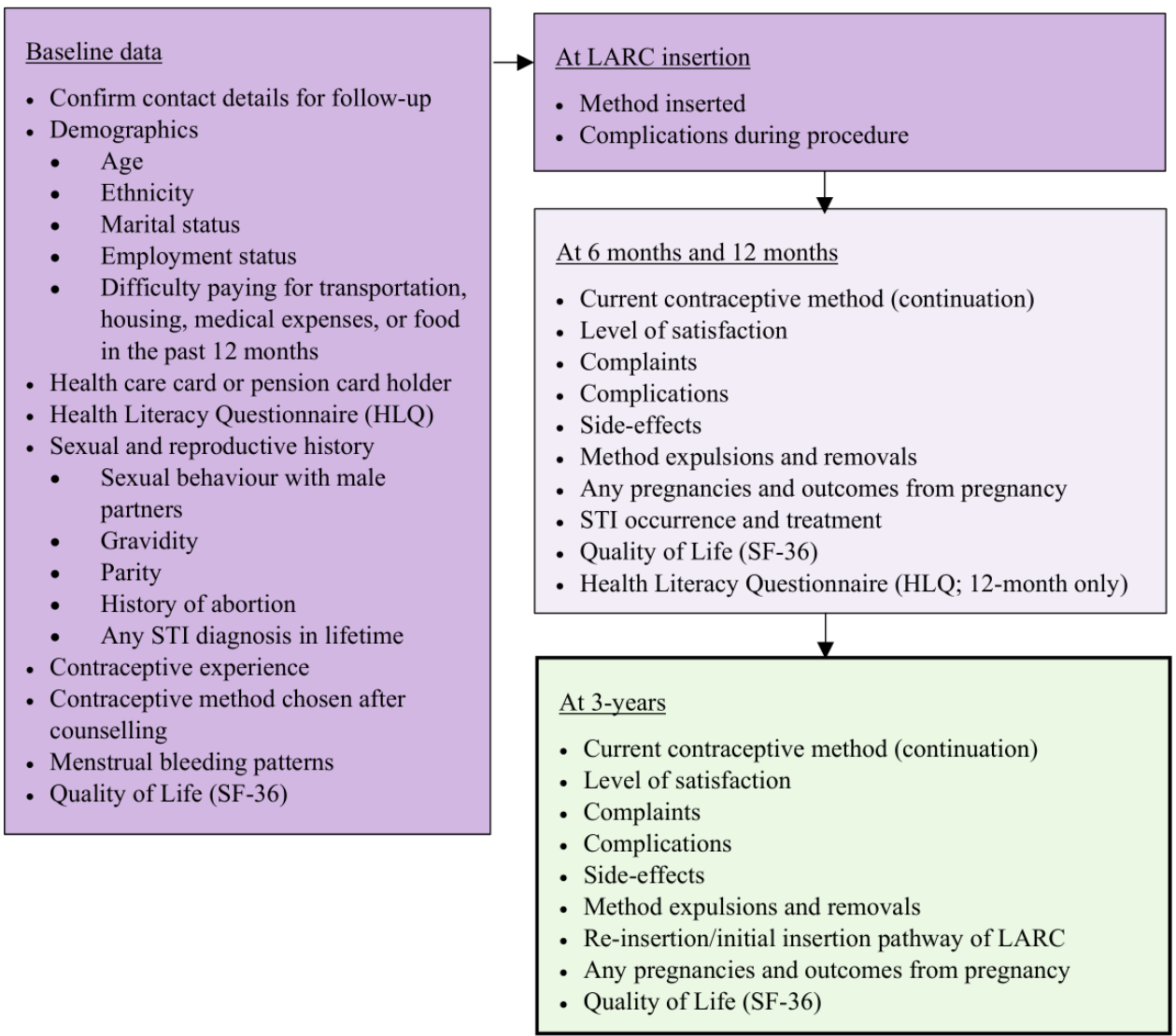

Figure 1 Variables that were recorded in the initial phase of the study and those that will be recorded for the 3-year longitudinal follow-up. LARC, long-acting reversible contraception; STI, Sexually Transmitted Infection.

\section{Outcome evaluation}

The association between contraceptive chosen (LARC or other method) on the continued use of that contraceptive method will be assessed using binary regression models with generalised estimating equations and robust standard errors to account for clustering. This analysis will allow adjustment for the group to which women were randomised as well as sociodemographic and reproductive history covariates, which will be necessary if there are differences between the characteristics of women who chose an LARC or other method during the study. This approach will also be used to assess which of the study (randomised group, contraceptive choice), sociodemographic (receiving public assistance or difficulty paying for basic necessities) and reproductive history covariates are associated with the other binary secondary outcomes (currently using an LARC; satisfied with contraceptive choice (scored $>2$ on 3-point Likert scale); unintended pregnancy since baseline; abortion since baseline). We will use multiple imputation and sensitivity analyses to explore the impact of missing data.

\section{Economic evaluation}

The direct costs of both the intervention and control arms will be measured as the costs of consultations with GPs and other medical practitioners and the costs of the contraceptive products, including their administration, where appropriate. Analysis of participants' Medicare
(Medicare Benefits Scheme and Pharmaceutical Benefits Scheme; MBS and PBS) records will allow for an accurate estimate of medical service and pharmaceutical utilisation and the associated costs. Indirect costs will be measured in terms of travel time and time away from work for women.

The primary outcome for the trial-based CEA will be the number of QALYs gained. QALYs will be estimated using the SF6D, a classification for describing health derived from a selection of SF-36 items. It is composed of six multilevel dimensions.

The results of the primary CEA will be reported as the cost/QALY gained. Cost-effectiveness will also be reported in terms of cost/change in HRQoL (measured using the SF-36, ${ }^{12}$ number of LARC inserted and type of contraception chosen). All cost-effectiveness results will be presented as net costs and benefits for the intervention arm versus the control arm at 36 months.

The costs of each arm will consider any cost-savings due to avoided unintended pregnancies. Mean estimates of costs will be used and confidence intervals generated by boot-strapping the data. The robustness and validity of the CEA will be explored using sensitivity analysis.

A modelled analysis is required to take into account costs and outcomes beyond the period of the trial. This will be informed by the outcomes and resource use observed during the trial, and extrapolated using data from the literature and other published sources. In addition to 
estimating outcomes in terms of QALYs, this analysis will also be able to assess the impact of the intervention on longer term consequences such as unintended pregnancies avoided and abortions avoided. This analysis will enable account to be taken of the long-term impact on the resource use and costs associated with LARC (which are typically more expensive 'up-front' but much less expensive over time than SARC), as well as longer term impacts such as side effects, discontinuations in contraceptive use (including LARC) and pregnancy rates.

\section{Participant data and study management}

All participants in the ACCORd Study (GPs and women) were allocated a unique code. Survey data will be recorded in a REDCap Database, along with the data from the previous surveys. Only ACCORd investigators and the project team will have access to this database.

\section{Ethics and dissemination}

The initial phase of the ACCORd Study, including the recruitment and training of GPs and the recruitment and follow-up surveys of the women, received approval from the Monash University Human Research Ethics Committee: CF16/188-201000080. Approval of an ethics amendment to conduct this 3-year longitudinal follow-up survey was also received.

The trial follow-up outcomes will be disseminated through formal academic pathways, including journal articles, national and international conferences and reports as well as using more 'mainstream' strategies such as seminars, workshops and media engagement. Additionally, outcomes will be communicated through policy briefs to Australian state and federal governments. Participants will be deidentified for the dissemination of the results.

\section{DISCUSSION}

Effective contraception is important in the prevention of unintended pregnancies. In Australia, the rates of unintended pregnancies are high, with a national population survey finding one in four respondents experienced an unintended pregnancy in the past 10 years. ${ }^{13}$ Moreover, the rates of LARC use among Australian women are low; just $11 \%$ among contraceptive users. ${ }^{14}$ Given their effectiveness ${ }^{131516}$ and high rates of user satisfaction and continuation, ${ }^{517}$ increasing the uptake of LARCs among Australian women has been identified as a priority by the Australian Healthcare and Hospitals Association. ${ }^{1}$

The ACCORd intervention addressed a number of barriers to LARC uptake in Australia; encouraging women to make better informed decisions around their contraceptive choice and enabling timely insertion for women who chose an LARC method. The ACCORd intervention resulted in an increased uptake of LARCs in the short term. Outcomes from the US CHOICE Study provide evidence of the long-term effectiveness of an intervention for increasing LARC uptake and it is, similarly, important to understand whether the effectiveness of the ACCORd intervention has been sustained over time.

Comparisons of continuation rates of contraceptive method chosen between intervention and control groups and a comparison of the number of incidents of unintended pregnancy will highlight the importance of providing appropriate training to GPs, who are ideally placed to provide contraceptive counselling for women and encourage sustainable and effective contraceptive use.

In addition, an economic evaluation of the study will be conducted to determine the cost-effectiveness of the intervention. Little is currently known about the economic cost of pregnancy or the cost-effectiveness of LARC in an Australian context. Recent analysis of international work on the cost-effectiveness of LARCs has assessed the generalisability of these study findings to the Australian context, suggesting that LARCs are likely to be a cost-effective option within the Australian context. ${ }^{18}$ In the US CHOICE Study, despite the extra cost of free LARC provision for participants, an increased uptake of LARC resulted in considerable cost-savings related to the avoidance of unintended pregnancies. ${ }^{19}$ The economic evaluation of the ACCORd Study will consider the cost structure of the Australian health system, including reimbursement by the MBS and PBS and out-of-pocket costs to women. The economic evaluation will highlight whether the burden of cost falls to the individuals or to the healthcare system.

The longitudinal results of this follow-up survey, including the CEA, will help to further establish the value and advantages of interventions aimed at educating GPs in effectiveness-based counselling and providing timely access to LARC insertion clinics. Findings will make important recommendations to improve the current Australian system of LARC provision. Moreover, the results will contribute to the body of work, both nationally and internationally, surrounding interventions and strategies to increase the uptake of LARCs and reduce global rates of unintended pregnancy.

Author affiliations

${ }^{1}$ Department of General Practice, Monash University, Melbourne, Victoria, Australia ${ }^{2}$ School of Public Health, The University of Sydney, Sydney, New South Wales, Australia

${ }^{3}$ Centre for Health Economics Research \& Evaluation, University of Technology Sydney, Sydney, New South Wales, Australia

${ }^{4}$ Department of Obstetrics \& Gynecology, Indiana University School of Medicine, Indianapolis, Indiana, USA

${ }^{5}$ School of Psychology and Public Health, La Trobe University, Melbourne, Victoria, Australia

${ }^{6}$ School of Public Health, University of Queensland, Brisbane, Queensland, Australia ${ }^{7}$ Judith Lumley Centre, La Trobe University, Melbourne, Victoria, Australia

${ }^{8}$ Family Planning Victoria, Melbourne, Victoria, Australia

${ }^{9}$ Royal Prince Alfred Hospital, The University of Sydney, Sydney, New South Wales, Australia

Contributors DM has led the development and conduct of the Australian Contraceptive ChOice pRoject Study and the concept of the longitudinal follow-up. NA has developed the protocol for the longitudinal follow-up survey. DM, CJW, KB, $\mathrm{AT}, \mathrm{JL}, \mathrm{KM}, \mathrm{MH}$ and JP were involved in the development of the protocol and design. 
KMcG provided support for the development of the statistical analyses plan. MH provided support for the development of the economic analyses plan. DM, CJW, JP, $A T$, JL, KMcG, MH, KM and KB edited and approved the final manuscript.

Funding This work was supported by the National Health and Medical Research Council grant ID: 1081743

Competing interests No competing interests.

Patient consent for publication Not required.

Provenance and peer review Not commissioned; externally peer reviewed.

Open access This is an open access article distributed in accordance with the Creative Commons Attribution Non Commercial (CC BY-NC 4.0) license, which permits others to distribute, remix, adapt, build upon this work non-commercially, and license their derivative works on different terms, provided the original work is properly cited, appropriate credit is given, any changes made indicated, and the use is non-commercial. See: http://creativecommons.org/licenses/by-nc/4.0/.

ORCID iDs

Natalie Amos http://orcid.org/0000-0001-6558-2580

Cathy J Watson http://orcid.org/0000-0002-2816-6956

\section{REFERENCES}

1 AHHA. Consensus statement: reducing unintended pregnancy for Australian women through increased access to long-acting reversible contraceptive methods. Australian Healthcare and Hospitals Association, 2017.

2 Mazza D, Watson CJ, Taft A, et al. Increasing long-acting reversible contraceptives: the Australian contraceptive choice pRoject (Accord) cluster randomized trial. Am J Obstet Gynecol 2020;222:S921. e1-S921.e13.

3 Mazza D, Black K, Taft A, et al. Increasing the uptake of longacting reversible contraception in general practice: the Australian contraceptive choice pRoject (Accord) cluster randomised controlled trial protocol. BMJ Open 2016;6:e012491.

4 Secura GM, Allsworth JE, Madden T, et al. The contraceptive choice project: reducing barriers to long-acting reversible contraception. Am J Obstet Gynecol 2010;203:115.e1-115.e7.
5 O'neil-Callahan M, Peipert JF, Zhao Q, et al. Twenty-fourmonth continuation of reversible contraception. Obstet Gynecol 2013;122:1083-91.

6 Birgisson NE, Zhao Q, Secura GM, et al. Preventing unintended pregnancy: the contraceptive choice project in review. J Womens Health 2015;24:349-53.

7 Diedrich JT, Zhao Q, Madden T, et al. Three-Year continuation of reversible contraception. Am J Obstet Gynecol 2015;213:662. e1-662.e8.

8 Madden T, Mullersman JL, Omvig KJ, et al. Structured contraceptive counseling provided by the contraceptive choice project. Contraception 2013;88:243-9.

9 RACGP. Guidelines for preventive activities in general practice. 9th ed. RACGP, 2016.

10 Osborne RH, Batterham RW, Elsworth GR, et al. The grounded psychometric development and initial validation of the health literacy questionnaire (HLQ). BMC Public Health 2013;13:658.

11 Ware JE, Sherbourne CD. The mos 36-item short-form health survey (SF-36). I. conceptual framework and item selection. Med Care 1992;30:473-83.

12 Brazier J, Roberts J, Deverill M. The estimation of a preferencebased measure of health from the SF-36. $J$ Health Econ 2002;21:271-92.

13 Taft AJ, Shankar M, Black Kl, et al. Unintended and unwanted pregnancy in Australia: a cross-sectional, National random telephone survey of prevalence and outcomes. Med J Aust 2018;209:407-8.

14 Richters J, Fitzadam S, Yeung A, et al. Contraceptive practices among women: the second Australian study of health and relationships. Contraception 2016:94:548-55.

15 Winner B, Peipert JF, Zhao Q, et al. Effectiveness of long-acting reversible contraception. N Engl J Med 2012;366:1998-2007.

16 Trussell J. Understanding contraceptive failure. Best Pract Res Clin Obstet Gynaecol 2009;23:199-209.

17 Stombaugh S, Whiteley M. In adolescent girls using contraceptives, do long-acting reversible contraceptives (LARC) have a higher rate of continuation than other forms of contraception? Evidence-Based Practice 2018;21.

18 Lynch M, De Abreu Lourenco R, Flattery M, et al. Reviewing the costeffectiveness of long-acting reversible contraceptive methods in an Australian context. Aust N Z J Obstet Gynaecol 2019;59:21-35.

19 Madden T, Barker AR, Huntzberry K, et al. Medicaid savings from the contraceptive choice project: a cost-savings analysis. Am J Obstet Gynecol 2018;219:595.e1-595.e11. 